\title{
Description de Plasmodium aegyptensis n. sp. parasite présumé du Muridé Arvicantbis niloticus en Haute-Égypte *
}

\author{
par GALAL AHMED ABD-EL-AZIZ *, Irène LANDAU ** \\ et François MILTGEN * * \\ * Faculté de Médecine, Assiut, Egypte. \\ ** Laboratoire de Zoologie (Vers), associé au C.N.R.S. \\ Muséum national d'Histoire naturelle, 43, rue Cuvier, F 75231 Paris Cedex 05.
}

\section{Résumé.}

Plasmodium aegyptensis n. sp. est proche de P. vinckei Rodhain, 1952 ; il s'en différencie par sa plus petite taille, sa forme compacte, son pigment plus abondant et plus grossier, et le nombre plus faible de mérozoïtes dans les rosaces.

\section{Summary.}

Description of Plasmodium aegyptensis, a parasite of the Muridae Arvicanthis niloticus in Upper Egypt.

Plasmodium aegyptensis n. sp. has some similarities with P. vinckei Rodhain, 1952. It differs from the latter by it's smaller size, it's compact and not amiboid shape, it's coarser and more abundant pigment and the smaller number of merozoites in the schizonts.

Le sang de 600 Muridés capturés dans la région d'Assiut sur la rive Ouest du Nil, à $375 \mathrm{~km}$ au Sud du Caire, a été examiné au cours des années 1972 et 1973 . Il s'agissait de 300 Arvicanthis niloticus (Desmarets) et de 300 Rattus norvegicus (Berkenhout), Rattus alexandrinus (Is. Geoffroy) et Acomys sp. La région, de climat et végétation semi-désertiques, est une oasis fortement cultivée (arbres fruitiers essentiellement).

* Travail effectué grâce à une subvention de l'Organisation Mondiale de la Santé. 
Parmi les lames de sang examinées, se trouvait un frottis étiqueté «Arvicanthis 》, avec une forte parasitémie par un Plasmodium et quelques Trypanosomes de type lewisi. Le Plasmodium diffère par sa morphologie de tous les autres Plasmodium de Mammifères connus en Afrique, et nous le décrirons ici comme espèce nouvelle sous le nom de Plasmodium aegyptensis n. sp.

L'origine de ce parasite reste cependant mystérieuse. De nombreux Arvicanthis ont été examinés auparavant en Egypte par des chercheurs exercés qui n'ont signalé aucun Plasmodium. Par ailleurs, une mission a été effectuée à Assiut en avril 1975 par l'un d'entre nous (F. Miltgen) et Killick-Kendrick. 20 Arvicanthis ont été capturés et splénectomisés sans qu'aucune parasitémie ne s'ensuive. Nous avons également éliminé une contamination accidentelle par $P$. berghei d'un Arvicanthis maintenu au laboratoire. En effet, la morphologie de $P$. berghei chez l'Arvicanthis est très différente de celle de $P$. aegyptensis. Enfin, d'après les renseignements qui nous ont été donnés, $P$. vinckei n'a jamais été maintenu au laboratoire à Assiut.

\section{Description}

Nous ne possédons qu'un seul frottis, en assez mauvais état, de $P$. aegyptensis. Cependant, son abondance sur la lame, où plusieurs endroits sont bien étalés et colorés, a rendu possible son étude morphologique.

\section{1. - Cycle asexué.}

Le tout jeune anneau est déjà un parasite compact avec un noyau rond et unique et un cytoplasme bleu vif; la vacuole est très petite, souvent limitée à une petite bande claire autour du noyau.

Le pigment apparaît très tôt et on l'observe dans de nombreuses formes uninucléées, en granules de taille variable. Le trophozoïte en cours de croissance reste compact, rond, sans tendances amiboïdes. La vacuole grandit légèrement et le noyau se divise. Le pigment, d'abord uniformément réparti, a alors tendance à occuper des plages d'étendue limitée. Dans les schizontes immatures, il s'agglomère en 2 à 3 amas compacts de granules assez grossiers.

Le schizonte mûr contient le plus souvent 6 mérozoïtes, parfois 4 , très exceptionnellement 8. Ceux-ci ont une forme générale arrondie ou légèrement allongée. Le pigment forme une seule masse noire et dense, bien limitée.

Planche I: Plasmodium aegyptensis n. sp. dans le sang d'Arvicanthis niloticus: 1-2: jeunes anneaux ; 3 : trophozoïte âgé ; 4-5-6: schizontes immatures; 7-8: schizontes mûrs; 9 : hématie mûre polyparasitée; 10: macrogamétocyte; 11: réticulocyte polyparasité. 

et collaborateurs

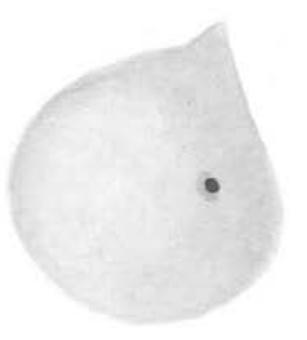

1

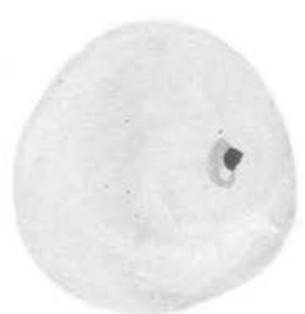

2

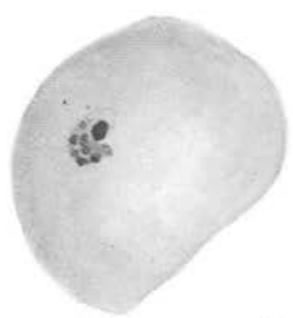

3
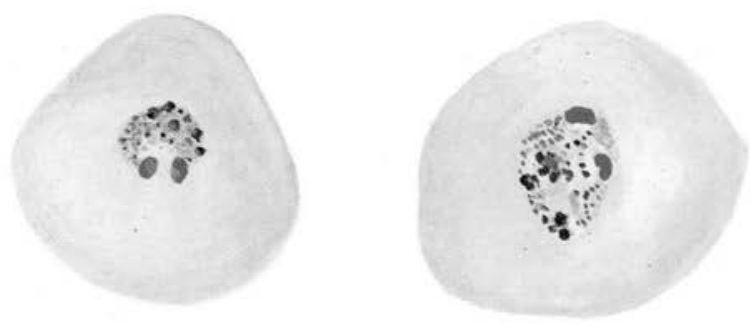

4
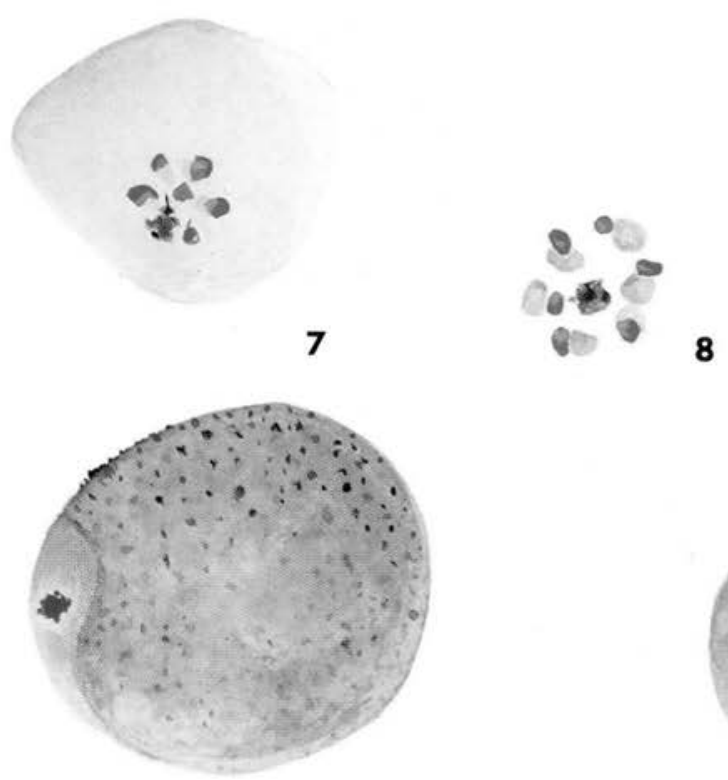

5

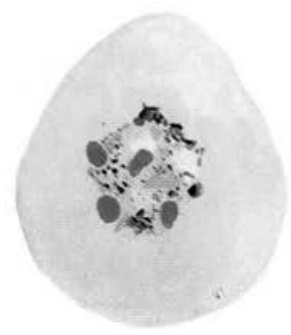

6

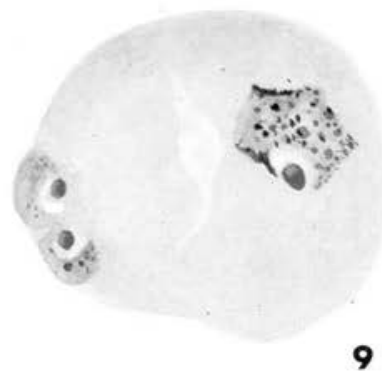

10

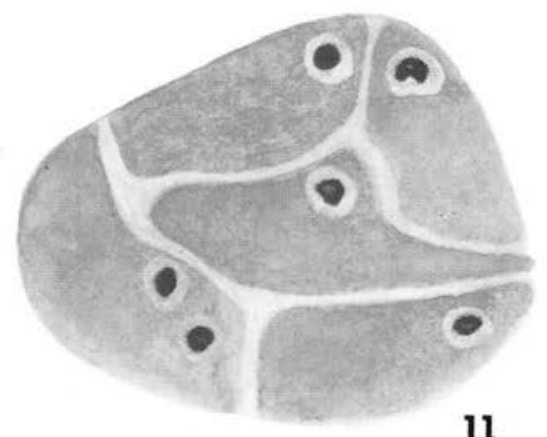

PLANCHE 1 

Quel que soit le stade observé, le parasite reste petit, occupant au maximum le $1 / 3 \mathrm{du}$ volume de la cellule-hôte. Le globule rouge parasité est généralement un érythrocyte mûr, dont la forme et la taille ne sont pas altérées. Cependant, le polyparasitisme d'une cellule n'est pas exceptionnel, et celle-ci peut alors être augmentée de taille. Quelques réticulocytes polyparasités ont été également observés : ils sont très hypertrophiés et contiennent jusqu'à 10 parasites, habituellement jeunes, et pour la plupart dépourvus de pigment.

\section{2. - Gamétocytes.}

Les gamétocytes immatures coiffent un pôle de l'hématie. A maturité, celle-ci a disparu et le gamétocyte a une taille légèrement supérieure à celle des globules voisins.

Le macrogamétocyte a un noyau arrondi formé de granules fins, siégant au centre d'une zone claire assez large et bien limitée. Le cytoplasme est bleu-gris et le pigment, fin, jaunâtre, laisse des plages non pigmentées, plus ou moins étendues, en bordure de la zone claire. Aucun microgamétocyte mûr n'a été observé.

\section{Discussion}

La présence d'infections mixtes par deux espèces de Plasmodium est une possibilité toujours présente à l'esprit lorsque l'on découvre un paludisme chez un petit Mammifère en Afrique. En effet, qu'il s'agisse de Thamnomys, de Chiroptères, ou d'Anomaluridés, 2 ou 3 espèces ont généralement été décrites pour chacun de ces hôtes.

Dans le cas de $P$. aegyptensis, il n'est pas possible d'affirmer avec certitude que les réticulocytes hypertrophiés et polyparasités par des trophozoïtes peu pigmentés n'hébergent pas, en fait, un Plasmodium de type berghei. Nous n'avons trouvé aucune autre forme pouvant être rattachée à une deuxième espèce, mais, bien que la présence de jeunes parasites dans les réticulocytes ne soit pas incompatible avec une espèce envahissant de préférence les hématies mûres, nous ne pouvons exclure complètement l'éventualité d'une infection double.

L'espèce la plus proche de P. aegyptensis est $P$. vinckei Rodhain, 1952 ; ces deux parasites ont en commun: la prédilection pour les hématies mûres, leur taille générale et l'aspect morphologique des schizontes.

$P$. aegyptensis diffère cependant de $P$. vinckei par de nombreux caractères; la vacuole des anneaux et des trophozoïtes est plus petite; les anneaux n'ont qu'une seule masse chromatinienne ; le pigment est plus abondant et plus grossier que celui de $P$. vinckei, sauf dans les gamétocytes, où il est au contraire plus fin et moins abondant; les rosaces de $P$. aegyptensis n'ont que 4 ou 6 mérozoïtes, ceux de $P$. vinckei, 
8 en moyenne, chez les souris blanches, beaucoup plus chez le Thamnomys; enfin, $P$. aegyptensis est dans l'ensemble plus petit et plus compact que $P$. vinckei.

Si la redécouverte de $P$. aegyptensis confirmait les renseignements que nous avons sur son origine, ce parasite prendrait un très grand intérêt: les Plasmodium de Rongeurs auraient un spectre d'hôtes et une distribution géographique plus étendus que l'on ne le pensait.

\section{Bibliographie}

RodhaIN (J.), 1952. - Plasmodium vinckei n. sp., un deuxième plasmodium parasite de Rongeurs sauvages au Katanga. Ann. Soc. belge Med. Trop., 32, 275-280. 